\title{
The role of gadoxetic acid-enhanced magnetic resonance cholangiography in the evaluation of postoperative bile duct injury: pictorial essay
}

O papel da colangiorressonância com ácido gadoxético na avaliação de complicações pós-cirúrgicas da via biliar: ensaio iconográfico

\section{Bruno Jucá Ribeiro ${ }^{1, a}$, Aldo Maurici Araújo Alves ${ }^{1, b}$, Rafael Santiago de Oliveira ${ }^{1, c}$, Fernanda Velloni ${ }^{1, d}$, Giuseppe D'Ippolito ${ }^{1, e}$}

1. Escola Paulista de Medicina da Universidade Federal de São Paulo (EPM-Unifesp), São Paulo, SP, Brazil.

Correspondence: Dr. Bruno Jucá Ribeiro. EPM-Unifesp - Departamento de Diagnóstico por Imagem. Rua Napoleão de Barros, 800 , Vila Clementino. São Paulo, SP, Brazil, 04024-002. Email: brunojucar123@gmail.com.

a. https://orcid.org/0000-0002-6947-1375; b. https://orcid.org/0000-0001-7310-5709; c. https://orcid.org/0000-0003-0838-3731; d. https://orcid.org/0000-0002-2019-7918; e. https://orcid.org/0000-0002-2701-1928.

Received 6 August 2018. Accepted after revision 13 September 2018.

How to cite this article:

Ribeiro BJ, Alves AMA, Oliveira RS, Velloni F, D'Ippolito G. The role of gadoxetic acid-enhanced magnetic resonance cholangiography in the evaluation of postoperative bile duct injury: pictorial essay. Radiol Bras. 2019 Nov/Dez;52(6):403-407.

Abstract latrogenic lesion of the bile ducts is a relatively common occurrence during liver surgery, increasing morbidity and mortality rates. T2-weighted magnetic resonance cholangiography and gadoxetic acid-enhanced functional magnetic resonance cholangiography ( $\mathrm{MMRC}$ ) with administration of hepatobiliary-specific contrast medium (gadoxetic acid) are fundamental to the diagnostic imaging approach in patients with such lesions. Here, we present a review of the literature and suggest an imaging approach to biliary tract injury, focusing on clinical cases in which fMRC had an impact on the decision-making process for the management of the affected patients.

Keywords: latrogenic disease; Bile ducts; Magnetic resonance imaging; Contrast media.

Resumo As lesões iatrogênicas das vias biliares são relativamente frequentes e aumentam as taxas de morbidade e mortalidade pósoperatória. A colangiografia por ressonância magnética ponderada em T2 e as imagens hepatobiliares funcionais com ácido gadoxético (CRM-F) são fundamentais na abordagem diagnóstica desses pacientes. Os autores apresentam uma revisão da literatura e sugerem uma manejo radiológico da lesão do trato biliar, demonstrando, por meio de casos clínicos, como a CRM-F pode contribuir no processo de tomada de decisão no manejo desses pacientes.

Unitermos: Doença iatrogênica; Vias biliares; Ressonância magnética; Meios de contraste.

\section{INTRODUCTION}

Iatrogenic lesion of the bile duct is a relatively common occurrence during liver surgery, increasing morbidity and mortality rates ${ }^{(1)}$. Biliary complications such as fistulas and stenoses occur after cholecystectomy in up to $0.6 \%$ of cases $^{(2)}$. In addition, patients submitted to biliary-enteric anastomosis are more likely to present complications (e.g., cholangitis and stones), which are estimated to occur in $10-30 \%$ of such patients ${ }^{(3,4)}$.

Laboratory tests or imaging examinations can be used in order to evaluate patients suspected of having an iatrogenic lesion of the biliary tract. Laboratory tests, notably quantification of alkaline phosphatase and gamma-glutamyl transferase, are sensitive but not very specific in the characterization of complications ${ }^{(5)}$.

As a general rule, ultrasound and computed tomography do not provide the necessary detail to define the appropriate treatment, being limited to the detection of bile duct dilatation and perihepatic fluid collections ${ }^{(1)}$.
The main disadvantages of endoscopic retrograde cholangiopancreatography (ERCP) and intraoperative cholangiography are their invasiveness, the difficulty in accessing the biliary tract in the presence of biliary-enteric anastomosis, and the high risk of complications, such as intraluminal bleeding and sepsis, as well as the difficulty to use ERCP in performing follow-up evaluations ${ }^{(5)}$. Therefore, magnetic resonance cholangiography (MRC) was established as an effective, noninvasive method for the detection of postoperative complications. By means of T2-weighted MRC (T2-MRC), it is possible to characterize stenoses and dilatations of the bile ducts, although T2-MRC does not provide functional information related to bile excretion and flow, which hinders the differentiation between mild and obstructive dilatation and the characterization of lesions in cases without dilatation ${ }^{(6,7)}$.

T1-weighted MRC with hepatobiliary-specific contrast (gadoxetic acid), also known as functional MRC (fMRC), is an emerging technique that has already proved 
useful in delineating the preoperative and postoperative anatomy of the biliary tree, as well as in detecting/characterizing biliary diseases and postoperative complications such as intraductal stones, stenoses, biliary fistulas, and cysts $^{(8,9)}$. In addition, it can provide functional information, which is useful for grading biliary stenosis, evaluating the dynamics of bile flow, and estimating the segmental liver function ${ }^{(10)}$. Various studies have demonstrated the usefulness of fMRC-based information on bile flow dynamics, some reporting rates of accuracy in the detection of postoperative lesions even higher than those obtained with $\mathrm{T} 2-\mathrm{MRC}^{(1)}$.

The objective of this essay was to present our experience with fMRC in the context of patients suspected of having postoperative complications of the bile ducts.

\section{PHARMACOKINETICS AND EXAMINATION PROTOCOL}

Gadoxetic acid is an ionic hepatobiliary-specific contrast agent composed of gadolinium and ethoxybenzyl-diethylenetriamine-pentaacetic acid (Gd-EOB-DTPA, Primovist; Bayer Schering Pharma, Berlin, Germany). In addition to the specific extracellular properties of the DTPA component, which allows image acquisition in the arterial, portal, and equilibrium phases, gadoxetic acid has lipophilic properties, provided by the EOB, which allows its gradual uptake ( $\leq 50 \%$ of the injected dose) by normally functioning hepatocytes. During the hepatobiliary phase, which begins, on average, 10-20 min after contrast injection in healthy individuals, it is possible to observe excretion of the contrast medium through the bile ducts, which provides enhancement of the biliary tree on T1-weighted sequences ${ }^{(10,11)}$.

The half-life of gadoxetic acid is approximately $2 \mathrm{~h}$, the peak of accumulation in hepatocytes occurring at 20-
$40 \mathrm{~min}$, hepatocyte uptake beginning at $3 \mathrm{~min}$, and biliary excretion beginning at $10 \mathrm{~min}$ (Figure 1). High levels of bilirubin (> $3 \mathrm{mg} / \mathrm{dL}$ ) and ferritin (i.e., hepatic dysfunction) can reduce contrast uptake by the liver, thereby delaying or reducing biliary excretion (Figure 2). Therefore, delayed image acquisition (at 20-180 min, in our experience) or a double dose of the contrast medium might be needed in order to achieve adequate enhancement of the biliary tree $\mathrm{e}^{(10,11)}$.

The fMRC protocol consists of three-dimensional T1-weighted sequences with fat saturation, axial and coronal images being acquired in the hepatobiliary phase (at 10, 20, and $40 \mathrm{~min}$ after injection of the contrast medium). Occasionally, it can be necessary to acquire images even 8-24 h later. At our facility, fMRC examinations are performed in one of two types of 3.0 T scanners: Achieva 3.0 T TX (Philips Medical Systems, Best, The Netherlands), with a repetition time of $1.83 \mathrm{~ms}$, echo time of $3.89 \mathrm{~ms}$, slice thickness of $3 \mathrm{~mm}$, flip angle of $30^{\circ}$, duration of $18 \mathrm{~s}$, and a matrix of $256 \times 134-\mathrm{or}$ Skyra 3.0 T (Siemens Healthcare, Erlangen, Germany), with a repetition time of $6.0 \mathrm{~ms}$, echo time of $2.46 \mathrm{~ms}$, slice thickness of $3 \mathrm{~mm}$, flip angle of $30^{\circ}$, duration of 18 $\mathrm{s}$, and a matrix of $256 \times 128$. The fMRC examinations are usually complemented with traditional sequences of the upper abdomen and T2-MRC.

\section{INDICATIONS}

\section{Stenosis}

T2-MRC has limitations in distinguishing the expected, slight patent narrowing in the plane of the biliary anastomosis, without clinical significance, from clinically relevant stenosis. In fMRC, gadoxetic acid provides functional information on biliary excretion in such a way

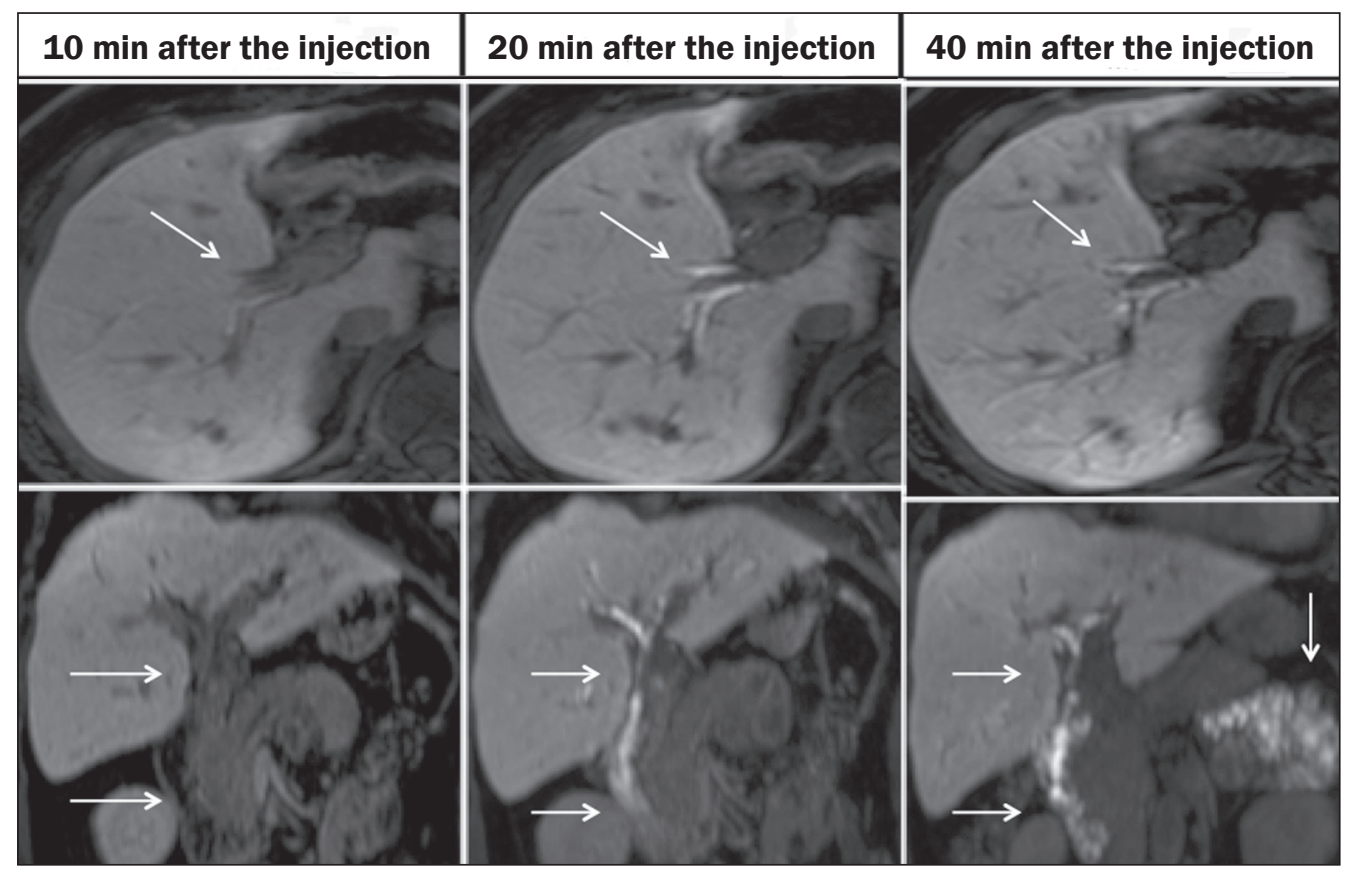

Figure 1. Representation of the normal time for excretion of contrast (gadoxetic acid) through the bile ducts in fMRC: 10,20 , and 40 min after the injection. 
Figure 2. Stenosis of the intrahepatic biliary tract after cholecystectomy. Axial T1-weighted fMRC sequences acquired at $20 \mathrm{~min}(\mathbf{A})$ and $4 \mathrm{~h}(\mathbf{B})$ after the injection of gadoxetic acid, showing moderate dilatation of the right biliary tract caused by stenosis in the plane of the right bile duct (arrow in $\mathbf{B}$ ). Note that the concentration of contrast medium is higher in the parenchyma of the left hepatic lobe (asterisk in $\mathbf{A}$ ) than in that of the right lobe, where there is a delay in the enhancement of the bile ducts (B). Diagnoses of thermal injury and bile duct ligation were considered.

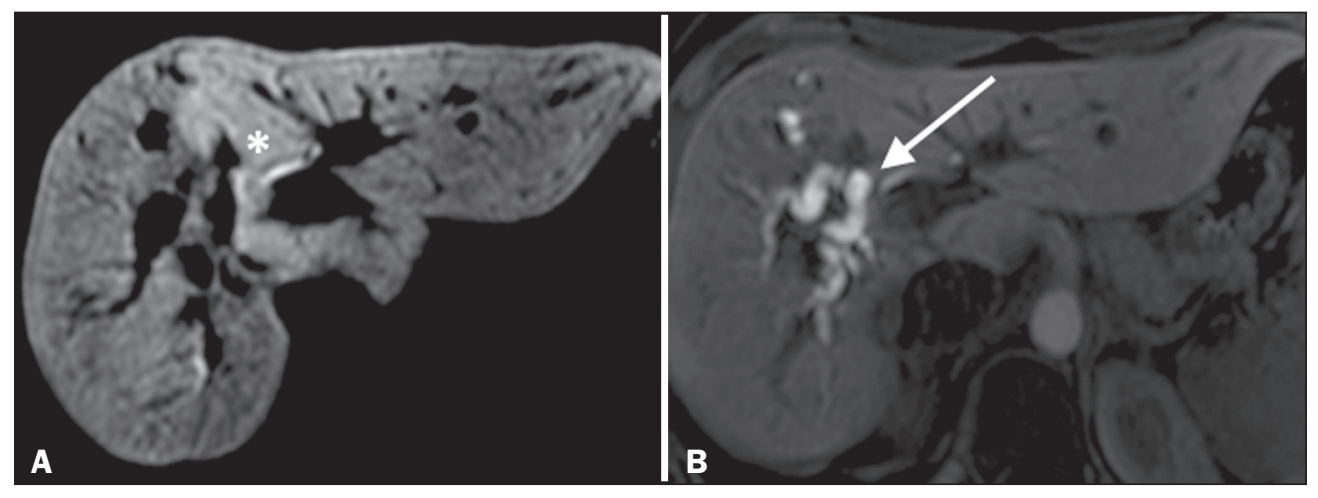

that a delay in the progression of the contrast medium at the suspected site is an important marker of significant stenosis, as is the absence of such progression. In our experience, as well as in other reports in the literature, the time to contrast filling of the biliary tract is directly proportional to the degree of stenosis, and acquisitions at up to $180 \mathrm{~min}$ after injection of the contrast medium are sometimes required (Figure 3). The opposite reasoning can be used in order to safely avert this complication; that is, the progression of the contrast medium through the stricture within a period of time considered physiological $(<20 \mathrm{~min}$; Figure 4) suggests that the narrowing (Figure $5)$ is not clinically significant ${ }^{(1,10)}$.

\section{Biliary fistula}

Fluid collections near or within the surgical field are common findings, especially in the context of major surgery, and are often irrelevant. However, in situations in which the biliary tract has been manipulated, biliary fistula emerges as an alarming possibility. Although T2-MRC sequences accurately detect fluid collections within the surgical field, they are of limited utility in identifying communication with the biliary tree, because, on T2-weighted images, communications present the same high signal intensity as does the biliary tree itself. Through the use of fMRC, we are able not only to identify the location of the biliary fistula but also to characterize the extravasation

Figure 3. Stenosis of the biliary anastomosis after liver transplantation. Coronal T2-MRC and T1-weighted MRC (A and $\mathbf{B}$, respectively): stenosis of the biliary anastomosis with mild dilatation of the intrahepatic biliary tree. Coronal $\mathrm{FMRC}$ at $40 \mathrm{~min}$ after the injection of gadoxetic acid (C,D): stenosis $(\mathbf{C})$ and a delay in the progression of contrast to the duodenal arc (D). Obvious stenosis with a delay in the progression of the contrast medium through the biliary tract.

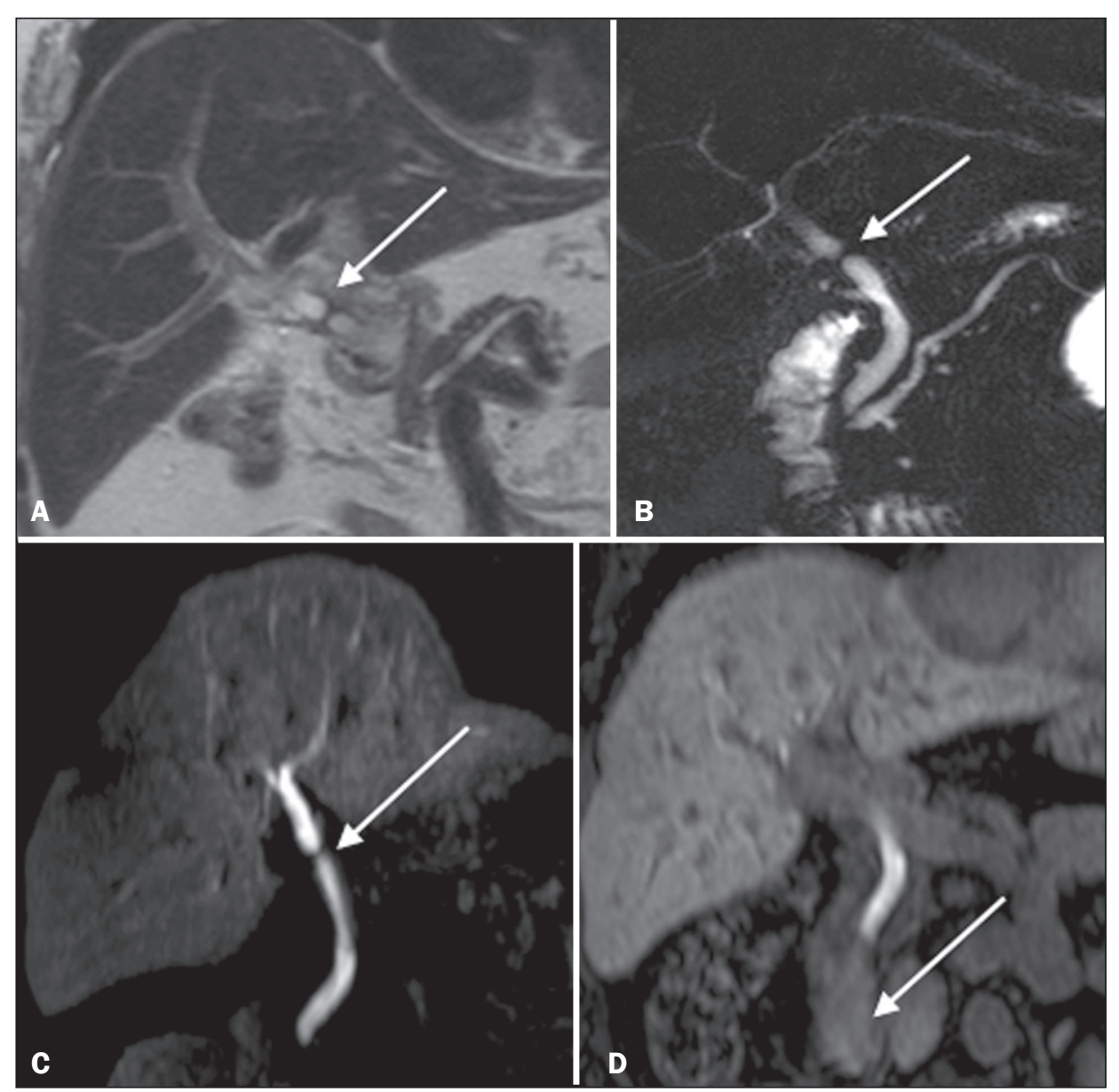



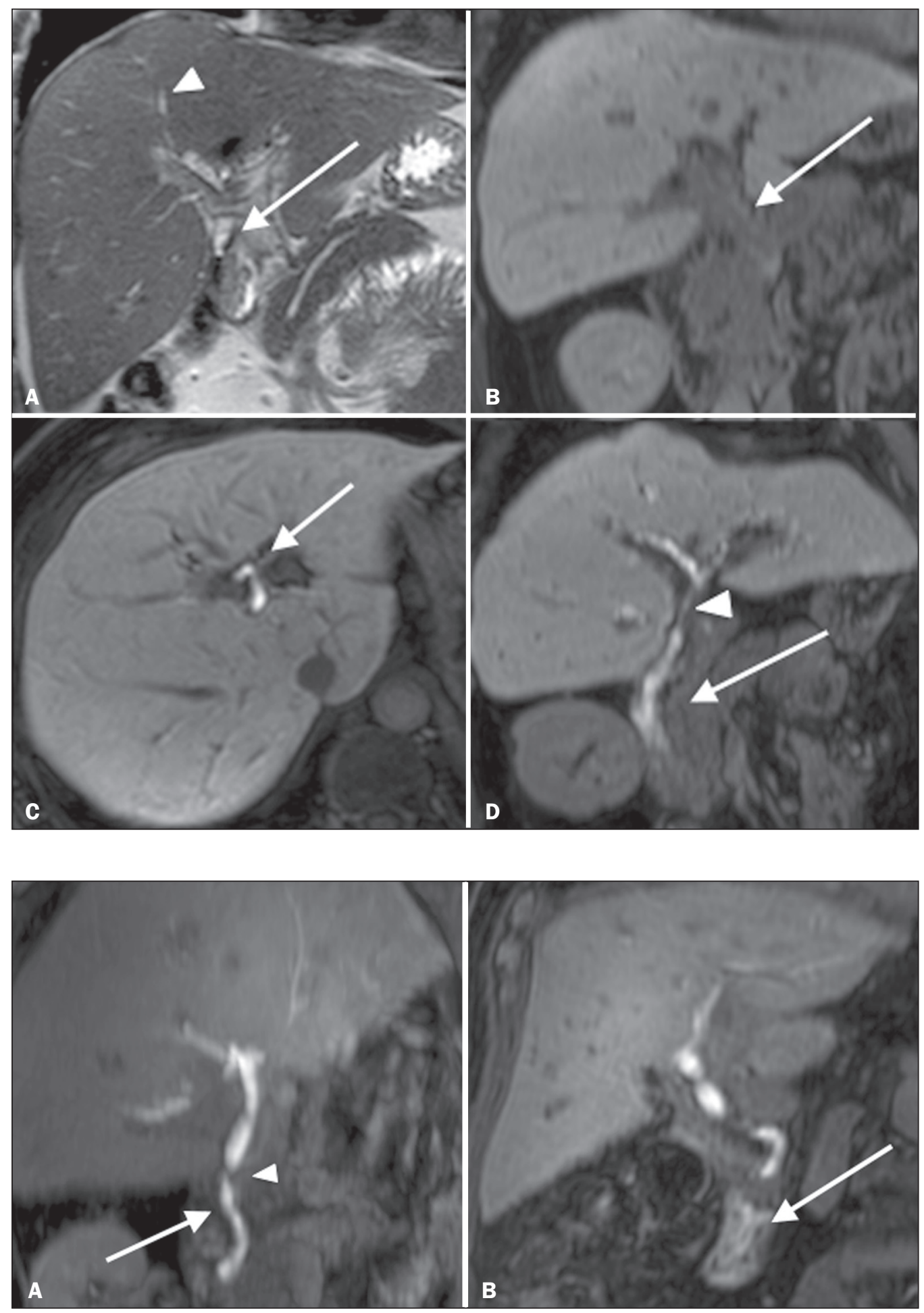

Figure 4. Evaluation of biliaryenteric anastomosis (after complicated cholecystectomy). Coronal T2-weighted sequence $(\mathbf{A})$, and T1 weighted fMRC sequences-axial (C) and coronal (B,D): biliary-enteric anastomosis and mild dilatation of the intrahepatic bile duct $10 \mathrm{~min}$ after injection of the contrast medium (arrow and arrowhead, respectively, in $\mathbf{A}$ ), and delayed excretion of contrast (B), which was not observed until 20 min after injection of the contrast medium (C,D). Patent stenosis of the biliary-enteric anastomosis with delayed biliary excretion. of contrast medium into the interior of a collection-unequivocal findings for the diagnosis of this complication (Figure 6). Therefore, fMRC provides information additional to that provided by T2-MRC, improving the identification and localization of biliary fistulas ${ }^{(12,13)}$.

\section{CONCLUSIONS}

For the characterization of stenoses and biliary fistulas, fMRC has proved to be a promising tool, in part due to its ability to provide information on bile flow dynamics. One drawback is the limited or insufficient excretion of hepatobiliary-specific contrast medium in patients with hepatic dysfunction and the longer duration of the examination. Nevertheless, fMRC has established itself as an important complement to T2-MRC, increasing accuracy in the detection of complications after surgical manipulation of the bile ducts. 
Figure 6. Biliary fistula after cholecystectomy. Unenhanced axial T1weighted sequence $(\mathbf{A})$ : no findings suggesting a lesion in the bile duct (asterisk). Axial and coronal fMRC (B and $\mathbf{C}$, respectively), $10 \mathrm{~min}$ after the injection of gadoxetic acid, showing the extravasation of contrast from the right bile duct to the abdominal cavity (arrows), which is consistent with biliary fistula.
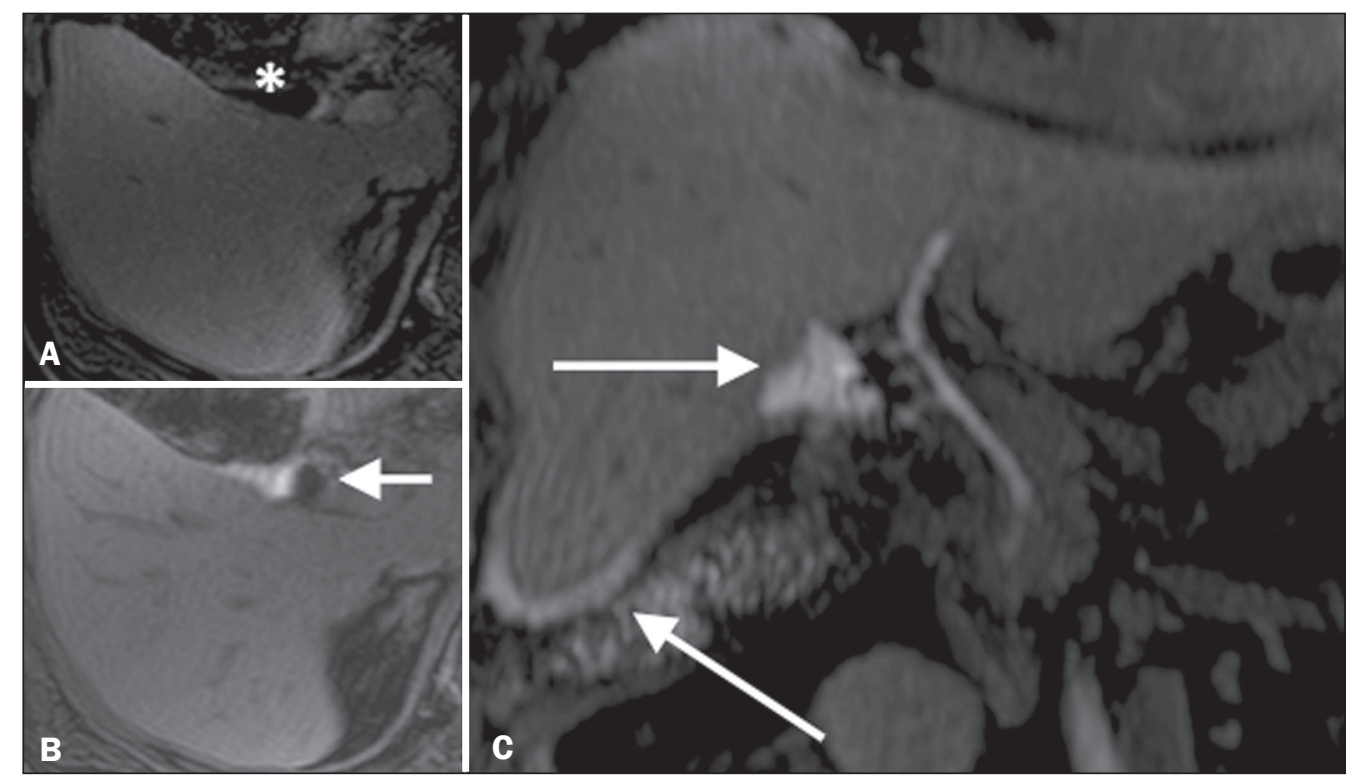

\section{REFERENCES}

1. Salvolini L, Urbinati C, Valeri G, et al. Contrast-enhanced MR cholangiography (MRCP) with GD-EOB-DTPA in evaluating biliary complications after surgery. Radiol Med. 2012;117:354-68.

2. Sicklick JK, Camp MS, Lillemoe KD, et al. Surgical management of bile duct injuries sustained during laparoscopic cholecystectomy: perioperative results in 200 patients. Ann Surg. 2005;241:786-92.

3. Nealon WH, Urrutia F. Long-term follow-up after bilioenteric anastomosis for benign bile duct stricture. Ann Surg. 1996;223:639-48.

4. Gruessner RWG, Benedetti E. Living donor organ transplantation. New York, NY: McGraw Hill; 2008.

5. Polistina FA, Frego M, Bisello M, et al. Accuracy of magnetic resonance cholangiography compared to operative endoscopy in detecting biliary stones, a single center experience and review of literature. World J Radiol. 2015;7:70-8.

6. Kandasamy D, Sharma R, Seith Bhalla A, et al. MR evaluation of biliary-enteric anastomotic stricture: does contrast-enhanced T1W MRC provide additional information? Clin Res Hepatol Gastroenterol. 2011;35:563-71.

7. Zurstrassen CE, Bitencourt AGV, Guimaraes MD, et al. Percutaneous stent placement for the treatment of malignant biliary obstruction: nitinol versus elgiloy stents. Radiol Bras. 2017;50:97-102.

\section{$(c)) \mathrm{BY}$}

8. Salvadori PS, Torres US, D'Ippolito G, et al. Contrast-enhanced magnetic resonance cholangiography with gadoxetic-acid-disodium for the detection of biliary-cyst communication in Caroli disease. Gastroenterol Hepatol. 2016;39:669-70.

9. Zattar-Ramos LC, Bezerra RO, Siqueira LTB, et al. Hepatocytespecific contrast agent-enhanced magnetic resonance cholangiography: perioperative evaluation of the biliary tree. Radiol Bras. 2017;50:389-94.

10. Boraschi P, Donati F. Biliary-enteric anastomoses: spectrum of findings on Gd-EOB-DTPA-enhanced MR cholangiography. Abdom Imaging. 2013;38:1351-9.

11. D'Ippolito G. The role of MRI using liver-specific contrast agent in the assessment of focal liver lesion. Radiol Bras. 2014;47(5):vii-viii.

12. Kantarcı M, Pirimoglu B, Karabulut N, et al. Non-invasive detection of biliary leaks using Gd-EOB-DTPA-enhanced MR cholangiography: comparison with T2-weighted MR cholangiography. Eur Radiol. 2013;23:2713-22.

13. Ribeiro KCP, Guimarães JPO, Aidar LB, et al. Hemobilia in a patient with arteriobiliary fistula after liver contusion. Radiol Bras. 2018;51:413-4. 